\title{
Research Paper: Effect of Novel Spring Damper and Posterior Leaf Spring Ankle Foot Orthosis on the Vertical Component of Ground Reaction Forces During Walking in Patients With Drop Foot
}

\author{
Ensieh Pourhosaingholi' ${ }^{1}$ (D) , Hassan Saeedi' ${ }^{1}$ (D) , Mohammad Kamali' ${ }^{1}$ (D) \\ 1. Department of Orthotics and Prosthetics, School of Rehabilitation Sciences, Iran University of Medical Sciences, Tehran, Iran.
}

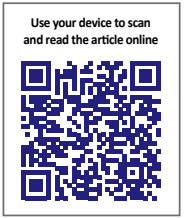

Crtation Pourhosaingholi E, Saeedi H, Kamali M. Effect of Novel Spring Damper and Posterior Leaf Spring Ankle Foo Orthosis on the Vertical Component of Ground Reaction Forces During Walking in Patients With Drop Foot. Journal of Research in Orthopedic Science. 2021; 8(1):17-22. http://dx.doi.org/10.32598/JROSJ.8.1.719.1

doi : http://dx.doi.org/10.32598/JROSJ.8.1.719.1

\section{(c) (1) (8)}

Article info:

Received: 10 Dec 2020

Revised: 15 Dec 2020

Accepted: 10 Jan 2021

Available Online: 01 February 2021

Keywords:

Orthotic device, Ankle foot orthosis, Gait, Ground reaction force, Brace, Gait, Rehabilitation

\begin{abstract}
A B S T RA C T
Background: Ankle Foot Orthoses (AFOs) are often prescribed in patients with drop foot. The purpose of this study was to investigate the effect of the novel designed storing-restoring hybrid passive AFO versus Posterior Leaf Spring (PLS) AFO on the peak and timing of vertical component of ground reaction force (vGRF) in patients with drop foot.

Objectives: the effect of novel designed storing-restoring hybrid passive AFO versus posterior leaf spring AFO on the peak and timing of Vertical Ground Reaction Force (vGRF) in drop foot patients.

Methods: Ten adults with drop foot ( 7 males and 3 females) were included in this study. Then, these patients walked at a self-selected speed with two AFOs. For each trial, the vGRF components were obtained using a Kistler force plate.

Results: the Independent t-test results showed a significant increase in the impact force in spring damper AFO than PLS $(\mathrm{P}<0.001)$. Significant differences were also found in the first and third peaks of vertical force and time of occurrence as well as the first minimum force and time of occurrence in spring damper than PLS AFO $(\mathrm{P}<0.001)$.

Conclusion: the novel AFO affects not only the impact force and peak of vGRF but also the timing of these forces. These changes indicate an improvement in the overall performance of the novel AFO.
\end{abstract}

\footnotetext{
* Corresponding Author:

Ensieh Pourhosaingholi, PhD.

Address: Department of Orthotics and Prosthetics, School of Rehabilitation Sciences, Iran University of Medical Sciences, Tehran, Iran.

Phone: + (918) 3399369

E-mail: ensiehpmd@yahoo.com
} 


\section{Introduction}

he current study exhibits a new insight into using a novel spring damper AFO;

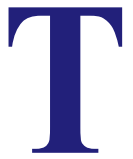
the impact loads, loading response load, and pre-swing phase force increased. Therefore, these findings showed that the use of this AFO may be useful for various drop foot patients as one of the key public health problems.

In drop foot disorder, ankle dorsiflexor muscles are weakened or paralyzed, leading to foot slap during the early step and the loss of ability to optimal toe clearance during the swing phase on the affected side [1-3]. As a result, some difficulties, like a decrease in walking speed, step length, and leg forward propulsion as well as changes in the Ground Reaction Force (GRF) pattern can be observed $[2,4-6]$.

Up to now, Ankle Foot Orthoses (AFOs), like posterior leaf spring AFOs, improve stability and toe clearance during the swing phase by fixing the ankle joint position $[2,3,7]$. However, these AFOs have some disadvantages, like limited movement of ankle joint, an increase in the knee flexion moment, and redirection of the vGRF pattern from normal condition [8]. In order to overcome some of these limitations and improve the biomechanical characteristics, several hybrid passive AFOs have been designed and fabricated with some extra elements than passive ones [9].

To our knowledge, limited information is available to support the hypothesis about the relationship between VGRF and different hybrid passive AFOs. In this regard, Amerinatanzi found that some hybrid passive articulate AFOs can improve vGRF pattern and peaks to near normal condition [4]. In another study, Rogozinski reported that AFOs with articulation may improve the vGRF vector by altering gait kinematic parameters at the initial contact [10]. However, some kinds of passive or stainless steel articulated hybrid passive AFOs deteriorated the pattern of vGRF with an extra peak at the mid-stance phase [4].

In our earlier studies, we designed and fabricated two novel AFOs and examined the effects of these AFOs on spatiotemporal parameters [11], kinetic and kinematic $[1,2]$, and balance-related parameters [1]. The purpose of this study was to investigate the effect of novel designed storing-restoring hybrid passive AFO versus posterior leaf spring AFO on the peak and timing of Vertical Ground Reaction Force (vGRF) in drop foot patients.

\section{Patients and Methods}

The study sample included ten right foot drop feet patients ( 7 men and 3 women) and all presented with unilateral drop foot, using PLS AFO as a routine AFO and ability to walk at least $20 \mathrm{~min}$ without assistance. Ethical approval was sought and obtained from the author's institute ethical approval board and prior to participation and all the patients filled up and signed an informed consent form before the study. The mean and Standard Deviation (SD) of age, weight, height, and body mass index of participants were $7.6(1.5)$ years, 65.18 (12.56) $\mathrm{kg}, 161.9(5.4) \mathrm{cm}$, and $21.40(1.60) \mathrm{kg} / \mathrm{m}^{2}$, respectively. These patients were randomly recruited from the Occupational Therapy Center, Rehabilitation School of Rehabilitation, Iran University of Medical Sciences [12].

\section{Intervention}

The spring damper AFO is an AFO with a storing restoring actuator weight of about $400 \mathrm{~g}$. This designed spring damper AFO composed of two separate carbon
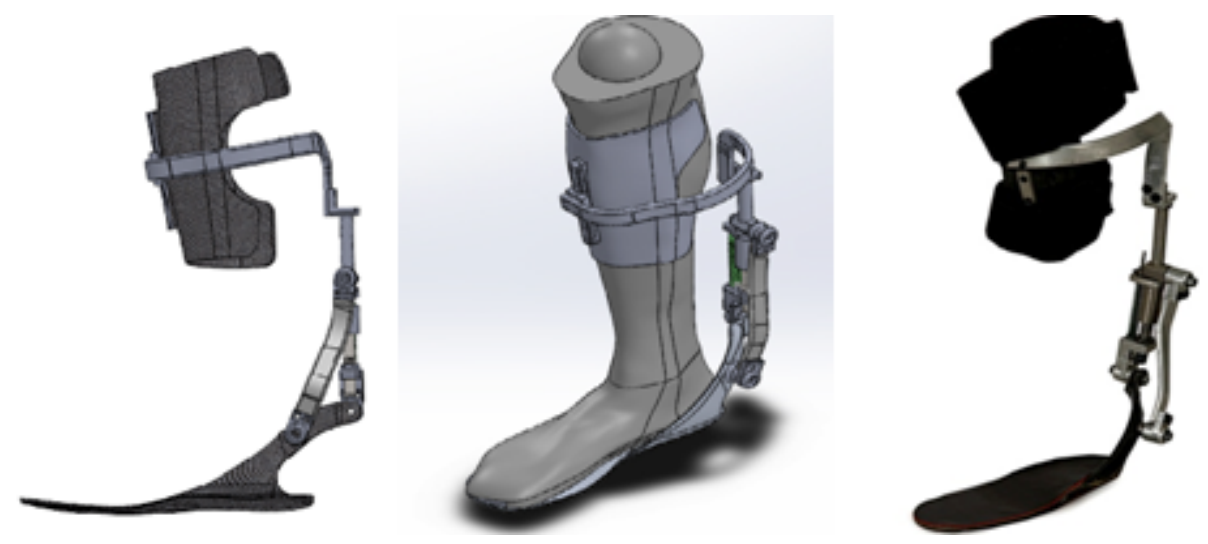

Figure 1. Novel spring damper Ankle Foot Orthosis (AFO)

Orthopedic Science 
composite sections, linked together by a sidebar to establish the attachment for actuator mechanism; however, other parts, including actuator connection and posterior hinge were made of titanium to decrease total AFO weight compared with the most existing hybrid passive AFOs $[1,2,6,13,14]$. The extensive description of the elements and function of this AFO was mentioned in our earlier studies (Figure 1).

\section{Instrument}

Force plates (Kistler Holding AG, Winterthur, Switzerland, Model 9286B), embedded into the floor were employed at a sampling rate of $1000 \mathrm{~Hz}$ [2] to determine the GRF.

\section{Procedure}

At the beginning of the examination, the process of trials was entirely explained to the participants. Then, they were asked to walk at a self-selected comfortable speed in the laboratory environment for at least 20 minutes to adapt with spring damper AFO prior to testing conditions. Before testing, the weight of each individual was determined using the force plate and the affected side data were extracted for the final analysis. Two conditions were tested: 1) walking with PLS, and 2) walking with spring damper AFO.

Patients were asked to walk (10 meters) at a self-selected comfortable speed and then rest for 2 minutes between each trial. The speed of walking was measured by a chronometer and trials of over 5\% speed difference were eliminated $[15,16]$. The patients walked two steps before reaching the force plate. The data were then collected from the five successful trials in each test condition.

\section{Data processing}

The primary variables of interest were as follows: the impact force at the initial contact (FZ0), the first peak of $\mathrm{vGRF}$ at the loading response (FZ1), the occurrence time of the first peak vGRF (T1), the minimum force of vGRF at the mid-stance (FZ2), the occurrence time of the minimum force vGRF (T2), the second peak of the vGRF at the pre-swing (FZ3), and the occurrence time of the second peak of the vGRF (T2).

We normalized the vGRF to the percentage of body weight and the entire stance cycle. The impact force was seen as a short spike of force (typically occurring within the first $50 \mathrm{~ms}$ ), superimposed on the upslope of the GRF, immediately following the initial contact of the foot $[17,18]$. Immediately, after the initial contact (within the first $50 \mathrm{~ms}$ ), the impact force was seen as a sudden spike, superimposed to the GRF curve. The stance phase was described as time from initial contact to toe-off phase [18]. GRFs data were collected using Qualysis software, transferred into the Excel software, extracted by MATLAB software, and finally analyzed by the SPSS v. 19 software, respectively.

\section{Statistics:}

SPSS Statistic 19.0 (SPSS, Inc., Chicago, IL, USA) was used for statistical analysis. The KolmogorovSmirnov test was used to demonstrate the normality of the variables. For evaluating the significance of GRF components in the two conditions of AFOs, the independent t-test was used and the significance level of $\alpha=0.05$.

\section{Results}

The results of this study are expressed as mean and standard deviation values of the peak force and timing of the vGRF in PLS AFO and novel AFO. The findings of the present study indicated that the novel designed AFO significantly increased vertical component and timing peak than the baseline PLS AFO in the first session and follow-up ( $\mathrm{P}<0.005)$ (Table 1).

\section{Discussions}

In this study, the vertical components of the GRF were assessed during walking in two conditions of using a spring-damper and PLS AFO. The vGRF pattern could be explained by considering the magnitude of the acceleration and applied force, arising from the weight of the body in the opposite direction of the ground force (Newton's second law) [19]. Based on this concept, different types of lower or assistive devices may produce different vGRF patterns [4].

In this study, while using spring damper AFO, the twopeak pattern of vGRF was near the normal condition. However, some studies have indicated that PLS or some conventional articulation AFOs with plantarflexion resistance were unable in restoring the normal pattern of vGRF [4]. In these AFOs, the peaks of vGRF at the early and terminal stance were decreased, and an extra peak was observed during the midstance. The probable cause of the appearance of this extra peak was structural rigidity or application of stiff elements, which impeded the normal GRF pattern or generated the high extra force and impulse [4]. 
Table 1. The Mean \pm SD of the peak force and timing in posterior leaf spring and spring damper Ankle Foot Orthosis

\begin{tabular}{|c|c|c|c|}
\hline \multirow{2}{*}{ Parameter Description } & \multicolumn{3}{|c|}{ Mean $\pm S D$} \\
\hline & Spring Damper AFO & PLS AFO & $\mathbf{P}$ \\
\hline Peak impact force at the initial contact & $45.85 \pm 5.4$ & $23.14 \pm 4.66$ & 0.00 \\
\hline Peak force at the loading response & $109.85 \pm 5.1$ & $94.63 \pm 10.8$ & 0.00 \\
\hline Time of force at the loading response & $15.11 \pm 0.48$ & $28.44 \pm 0.51$ & 0.00 \\
\hline Minimum force at the mid stance & $75.1 \pm 6.31$ & $104.23 \pm 1.32$ & 0.00 \\
\hline Time of force at the mid stance & $43.10 \pm 2.41$ & $55.36 \pm 2.36$ & 0.00 \\
\hline Peak force at the pre-swing & $110.24 \pm 1.53$ & $95.48 \pm 0.39$ & 0.04 \\
\hline Time of force at the pre-swing & $51.28 \pm 0.49$ & $62.9 \pm 0.29$ & 0.02 \\
\hline
\end{tabular}

Orthopedic Science

At initial contact, a significant increase was observed at the impact force (FZ0) using spring damper AFO than PLS. This increase was provided somewhat by increasing in the ankle overall Range of Motion (ROM), the shock absorption, stable foot landing, as well as gradual accepting the body weight in spring damper AFO [2]. However, it seems using PLS, structural rigidity, poor gait symmetry, and a decrease in push-off power [1] decreased the initial contact impact force.

During the loading response, a significant increase was observed in the peak force (FZ1) and a significant decrease was found in the occurrence time of this force (FZ1) using spring damper AFO than PLS. It seems that an adjustable damper element emulated the eccentric contraction of dorsiflexor muscles and provided controlled plantar flexion, without a remarkable reduction in plantar flexion ROM [2]. At this time, the entire body weight is rapidly moved the foot and the ground, the center of mass is moved downwards with some decelerations, and the inertial force is also added to the vertical component of GRF with the peak of approximately $107 \%$ of the body weight $[19,20]$. Possibly, the damper element of spring-damper AFO provided a controlled plantar flexion, which may help support the body mass and accelerate the center of mass. However, using PLS, the reduction in F1 may be the result of the absence of the eccentric contraction in dorsiflexion and AFO structural rigidity, preventing enough plantar flexion and leading to foot slap.

During the mid-stance, a significant decrease was observed at the peak FZ2 and the occurrence time of this force (TZ2) using spring damper AFO than PLS. During this phase, the posterior hinge as well as the frontal rail element showed an improvement toward propulsion to the dorsiflexion direction and helped the spring element store energy, which has been confirmed previously [2, 17, 21]. At this phase, the body starts to progress with the knee extending over the single limb, raising the center of mass until the highest point. Therefore, the GRF is less than $85 \%$ of the body weight as a result of a downward acceleration in the center of mass, which was added to the upward inertial force [19]. However, using PLS and some AFOs with stainless steel spring, an extra peak was observed. This extra peak was the result of structural rigidity or stiff spring leading to a decrease in the ankle ROM and normal acceleration of the $\operatorname{COM}[4,20]$.

During the pre-swing phase, a significant decrease was observed at the peak FZ3 and the occurrence time of this force (TZ3) using spring damper AFO than PLS. At this time, the co-activation of spring and damper elements released the stored energy from the mid-stance phases to generate the push-off power, which is in line with other studies $[2,17]$. In this phase, the heel lifts, and the foot is pushed down and back into the ground by plantar flexor muscle and the center of mass falls again. This downward deceleration, accompanied by the limb forward propulsion complex, caused the second peak of approximately 1.2 times of the person's body weight [20]. An increase in the peak GRF typically was associated with the forward progression of the weight and a raise in the center of mass [20]. using PLS, structural rigidity may hinder the forward progression of the tibia over the ankle foot complex. This drawback may lead to a decrease in the peak force as well as a poor ability to push off [2].

To our knowledge, no study has yet introduced hybrid passive AFOs, like spring damper with the ability to provide controlled plantar flexion at the loading response, free angular movement at the ankle joint during mid- 
stance, and improved push-off power at the pre-swing phase.

This study had some limitations: firstly, this study focused on the GRF without pay attention to the kinematic parameters and muscle electromyography; secondly, the sample size was small; thus, larger sample size and longterm daily use associated with gait training are needed; and finally, in this study, the immediate biomechanical effect of spring-damper AFO on vGRF was investigated and the longer-term effects are unknown.

\section{Conclusion}

Although AFOs prevented the ankle plantar flexion (plantarflexion resistance) at the swing phase to prevent toe dragging through the ground, they could not prevent foot slap. We employed damper and spring elements to improve the vGRF outcome measure to near the normal condition.

\section{Ethical Considerations}

\section{Compliance with ethical guidelines}

This study was approved by the Ethics Committee of the Iran University of Medical Sciences.

\section{Funding}

This research did not receive any grant from funding agencies in the public, commercial, or non-profit sectors.

\section{Authors' contributions}

All authors equally contributed to preparing this article.

\section{Conflict of interest}

The authors declared no conflict of interest.

\section{References}

[1] Pourhoseingholi E, Saeedi H, Kamali M, Jalali M. The effect of articulated $\mathrm{AFO}$ with Hydra pneumatic damper in biomechanical characteristic of drop foot. Med J Islam Repub Iran. 2020; 34(1):785-9. http:// mjiri.iums.ac.ir/article-1-5336-en.html

[2] Saeedi H, Pourhoseingholi E. Comparison the effect of kinetic parameters of innovative storing-restoring hybrid passive(comfort gait) ankle-foot orthosis(AFO) with posterior leaf spring AFO in drop-foot patients: A prospective cohort study. Curr Ortho Prac. 2020; 31(5):437-41. [DOI:10.1097/BCO.0000000000000915]
[3] Amerinatanzi A, Zamanian H, Shayesteh Moghaddam N, Jahadakbar A, Elahinia M. Application of the superelastic NiTi spring in Ankle Foot Orthosis (AFO) to create normal ankle joint behavior. Bioengineering (Basel). 2017; 4(4):95. [DOI:10.3390/bioengineering4040095] [PMID] [PMCID]

[4] Pourhosseingholi E, Farahmand B, Bagheri A, Kamali M, Saeb M. Efficacy of different techniques of AFO construction for hemiplegia patients: A systematic review. Med J Islam Repub Iran. 2019; 33:50. [DOI:10.47176/mjiri.33.50] [PMID] [PMCID]

[5] Alam M, Choudhury IA, Mamat AB. Mechanism and design analysis of articulated ankle foot orthoses for drop-foot. Sci World J. 2014; 2014:867869. [DOI:10.1155/2014/867869] [PMID] [PMCID]

[6] Mulroy SJ, Eberly VJ, Gronely JK, Weiss W, Newsam CJ. Effect of AFO design on walking after stroke: Impact of ankle plantar flexion contracture. Prosthet Orthot Int. 2010 34(3):277-92. [DOI:10.3109/03093646.2010.501512] [PMID]

[7] Dollar AM, Herr H. Lower extremity exoskeletons and active orthoses: Challenges and state-of-the-art. IEEE Trans Robot. 2008; 24(1):144-58. [DOI:10.1109/TRO.2008.915453]

[8] Rogozinski BM, Schwab SE, Kesar TM. Effects of an articulated ankle foot orthosis on gait biomechanics in adolescents with traumatic brain injury: A case-series report. Phys Med Rehabil Int. 2018; 5(2):1144. [PMID] [PMCID]

[9] Saeedi H, Pourhoseingholi E, Kamali M. A Comparison the spatiotemporal efficiency of two designs of Posterior Leaf Spring (PLS) and pneumatic damper Ankle-Foot Orthoses (AFO) in drop foot. Func Disabil J. 2019; 2(1):171-6. http:/ / fdj.iums.ac.ir/article-1-92-en.html

[10] Pourhoseingholi E, Kamali M, Saeedi H, Jalali M. The comparison of the effect of innovative designed storing-restoring hybrid passive $\mathrm{AFO}$ versus posterior leaf spring AFO on ankle joint kinematic in drop foot patients: A case series using a single subject design. Med J Islam Repub Iran. 2020; 34(1):120815. http://mjiri.iums.ac.ir/article-1-5910-en.html

[11] Pourhoseingholi E, Saeedi H. Role of the newly designed hybrid passive ankle-foot orthosis on self-reported balance confidence in post stroke drop foot. J Bodywork Movement Ther. 2020; 26:501-5. [DOI:10.1016/j.jbmt.2020.11.022]

[12] Creaby MW, May K, Bennell KL. Insole effects on impact loading during walking. Ergonomics. 2011; 54(7):665-71. [D OI:10.1080/00140139.2011.592600] [PMID]

[13] Murley GS, Landorf KB, Menz HB. Do foot orthoses change lower limb muscle activity in flat-arched feet towards a pattern observed in normal-arched feet? Clin Biomech (Bristol, Avon). 2010; 25(7):728-36. [DOI:10.1016/j.clinbiomech.2010.05.001] [PMID]

[14] Marasović T, Cecić M, Zanchi V. Analysis and interpretation of ground reaction forces in normal gait. WSEAS Trans Syst. 2009; 8(9):1105-14. https://www.researchgate.net/ publication/255602319_Analysis_and_Interpretation_of_ Ground_Reaction_Forces_in_Normal_Gait

[15] Daryabor A, Saeedi H, Ghasemi MS, Yazdani M, Kamali M, Nabavi $\mathrm{H}$, et al. Influence of heel design in an orthopedic shoe on ground reaction forces during walking. Prosthet Orthot Int. 2016; 40(5):598-605. [DOI:10.1177/0309364615596065] [PMID]

[16] Barela AM, Freitas PBd, Celestino ML, Camargo MR, Barela JA. Ground reaction forces during level ground walking with 
body weight unloading. Braz J Phys Ther. 2014; 18(6):572-9. [DOI:10.1590/bjpt-rbf.2014.0058] [PMID] [PMCID]

[17] Kitaoka HB, Crevoisier XM, Harbst K, Hansen D, Kotajarvi B, Kaufman K. The effect of custom-made braces for the ankle and hindfoot on ankle and foot kinematics and ground reaction forces. Arch Phys Med Rehabil. 2006; 87(1):130-5. [DOI:10.1016/j.apmr.2005.08.120] [PMID]

[18] Pittaccio S, Viscuso S, Beretta E, Turconi AC, Strazzer S. Pilot studies suggesting new applications of NiTi in dynamic orthoses for the ankle joint. Prosthet Orthot Int. 2010; 34(3):305-18. [DOI:10.3109/03093641003702253] [PMID]

[19] Peterson CL, Cheng J, Kautz SA, Neptune RR. Leg extension is an important predictor of paretic leg propulsion in hemiparetic walking. Gait Posture. 2010; 32(4):451-6. [DOI:10.1016/j.gaitpost.2010.06.014] [PMID] [PMCID]

[20] Chung CL, DiAngelo DJ, Powell DW, Paquette MR. Biomechanical comparison of a new dynamic ankle orthosis to a standard ankle-foot orthosis during walking. J Biomech Eng. 2020; Online ahead of print. [DOI:10.1115/1.4045549] [PMID]

[21] Karatsidis A, Bellusci G, Schepers HM, De Zee M, Andersen MS, Veltink PH. Estimation of ground reaction forces and moments during gait using only inertial motion capture. Sensors. 2017; 17(1):75. [DOI:10.3390/s17010075] [PMID] [PMCID] 